\title{
Thermal properties of fiber Bragg gratings inscribed point-by-point by an infrared femtosecond laser
}

\author{
Amós Martinez, Igor Khrushchev, lan Bennion \\ Photonics Research Group, Aston University, Birmingham B4 7ET, UK
}

Direct, point-by-point inscription of fiber Bragg gratings by infrared femtosecond laser had been recently reported. Response of these gratings to annealing at temperatures in range $500^{\circ} \mathrm{C}$ to $1050^{\circ} \mathrm{C}$ is studied for the first time. Gratings inscribed by infrared femtosecond lasers were thermally stable at temperatures up to $900^{\circ} \mathrm{C}$, representing a significant improvement in comparison with the "common", UV-inscribed, gratings. Annealing at temperatures up to $700^{\circ} \mathrm{C}$ increased grating reflectivity.

OCIS codes: (350.2770) Gratings; (320.2250) Femtosecond phenomena

Introduction: Direct laser writing of fibre gratings by a focused femtosecond laser has been demonstrated, including directly written long period gratings [1], fiber Bragg gratings (FBG) produced using special phase-masks [2], and FBGs produced by a femtosecond UV laser using standard phase mask [3].

Recently, we reported a method for direct writing of FBG using point-by-point irradiation by an infrared femtosecond laser [4]. This method requires neither phase masks nor photosensitised fibers and hence offers a remarkable technological flexibility with a processing time of less than 60 second.per FBG. High quality, strong gratings were produced using this method in commercial, non-photosensitive fibers. Growing interest in femtosecond laser inscription is based, among other factors, on the fact that the physical mechanism of this process is essentially different to that of 
UV inscription. As a result, the physical properties of the femto-inscribed structures and devices are different to those of the UV-produced equivalents.

The refractive index change in femtosecond inscribed gratings is believed to be initiated by formation of localized plasma in the bulk of the material causing densification of the latter. The process involves highly nonlinear photo-ionization, thus requiring high intensity of light and it only occurs in a tightly focused laser beam. Previous reports indicate that; contrary to UV inscription, femto-inscription does not depend strongly on formation of defects [5]. As a result, the decay of defects caused by thermal annealing is likely to be insignificant in the femto-inscribed structures. Hence, femtosecond inscription should produce structures with improved thermal robustness compared to UV-written structures.

High thermal stability of the femto-inscribed structures has been observed recently in the FBGs produced by using a special, high-order phase mask [2]. In this paper, we present a study of thermal properties of FBGs produced using direct, point-by point inscription. These devices present smaller modified areas and higher refractive index contrast than gratings inscribed with a femtosecond laser and phase-mask. We established that these FBG are stable up to a temperature of $900^{\circ} \mathrm{C}$. Annealing at temperatures up to $700^{\circ} \mathrm{C}$ actually increased the grating reflectivity, indicating the relaxation of strains induced during inscription in the vicinity of the modified region.

Experiment. Experimental setup and inscription procedure were similar to those used in our earlier study [3]. An amplified laser system, operating at a wavelength of $800 \mathrm{~nm}$, was used. The laser produced 150fs-long pulses at a repetition rate of 1 $\mathrm{KHz}$. The beam was focused into the fiber core by a X100 microscopic objective. Standard fibre (SMF) was used; no photosensitization procedure was carried out prior to the exposure. 
Pulse energy was set to approximately $0.5 \mu \mathrm{J}$ corresponding to the peak intensities in the order of $10^{14} \mathrm{~W} / \mathrm{cm}^{2}$. The gratings were approximately $30 \mathrm{~mm}$ long.

The pitch size in the gratings used in this work was $1.07 \mu \mathrm{m}$, corresponding to the second order of the Bragg reflection at a wavelength of $1.55 \mu \mathrm{m}$. Typical spectral characteristics of a femto-inscribed FBG are presented in figure 1, and are consistent with those reported previously [3].

Three similar samples of the femto-inscribed FBGs with reflectivity ranging from $80 \%$ to $90 \%$ were placed in an oven and annealed at constant temperatures of $500^{\circ} \mathrm{C}$, $700^{\circ} \mathrm{C}$ and $1000^{\circ} \mathrm{C}$, respectively, for a period of 24 hours. The grating spectra were monitored every 30 minutes by an analyzer with a resolution of $5 \mathrm{pm}$. After the annealing period, the oven was switched off and the gratings were allowed to cool down to room temperature. Monitoring of the grating spectra with the 30 -minute intervals was continued during the first 10 hours of the cooling process.

Three UV inscribed FBGs were used as control samples. These were inscribed in hydrogenated fiber by using a $90 \mathrm{~mW}$ beam from a CW laser operating at a wavelength of $244 \mathrm{~nm}$. After inscription, the FBGs were post-processed by annealing at $80^{\circ} \mathrm{C}$ for 24 hours. The resultant reflectivity of control samples before the tests was in excess of $98 \%$. The three control samples were subjected to the procedures of annealing and measurement identical to the ones applied to the femto-inscribed samples as described above.

Results. Measured evolution of the grating reflection is presented in figure 2 . Femtosecond inscribed gratings showed a significantly improved thermal stability compared to the hydrogenated, UV inscribed FBGs. The UV inscribed sampleexperienced a significant degradation at $500^{\circ} \mathrm{C}$ and was rapidly erased at $700^{\circ} \mathrm{C}$, whilst the femto-inscribed FBG did not show any signs of rapid decay up to 
$1000^{\circ} \mathrm{C}$. Comparison with the similar studies from literature shows that the thermal stability of the femto-inscribed FBGs is better than that of common Type I and Type IIA gratings and is similar to the stability of the Type II gratings, based on optical damage [4].

In all cases, we observed that the grating reflectivity after cooling down to room temperature was greater than that at the corresponding high temperature. This could be explained by relaxation of mechanical stress created in the outer regions of the modified area. Similar annealing behaviour has been previously observed femtoinscribed photonic crystals [6]. At lower temperature levels of $500^{\circ} \mathrm{C}$ and $700^{\circ} \mathrm{C}$, this effect dominated in femto-inscribed gratings and the resulting reflectivity actually increased after the annealing-cooling cycle in those gratings.

Fig.3. shows the dynamics of grating spectra during the cooling down period. The measurements were taken after a 24 hour - annealing at $700^{\circ} \mathrm{C}$. The measured spectral shift of $12.8 \mathrm{pm} /{ }^{\circ} \mathrm{C}$ is in line with the values of $13 \mathrm{pm} /{ }^{\circ} \mathrm{C}$ approximately, reported earlier for the UV-written FBGs [7].

In the next experiment, the same femto-inscribed grating was annealed successively at increasing temperatures of $500^{\circ} \mathrm{C}, 700^{\circ} \mathrm{C}, 900^{\circ} \mathrm{C}, 1000^{\circ} \mathrm{C}$ and $1050^{\circ} \mathrm{C}$. Annealing at each temperature lasted for 20 hours, after which the grating was allowed to cool down for 5 hours to room temperature before the next annealing cycle. The dynamics of the grating reflection during this exercise is shown in Figure 4.

Firstly, the grating reflection dropped during each heating period and subsequently increased during the cooling period, similarly to the behavior during the previous experiment as described above. Reflectivity decrease caused by annealing at lower temperatures was reversible. A certain increase in reflectivity was observed after the heating-cooling cycles at temperatures of $500^{\circ} \mathrm{C}$ and $700^{\circ} \mathrm{C}$. The $900^{\circ} \mathrm{C}$ cycle 
produced a slight overall decrease of reflectivity and, the $1000^{\circ} \mathrm{C}$ cycle caused a significant permanent degradation of the grating performance. Finally, annealing at a temperature of $1050^{\circ} \mathrm{C}$ practically erased the grating, rapidly and irreversibly reducing the reflection coefficient to a level below $20 \%$.

Overall, the results in this work are complementary to those reported in previous studies of the thermal behavior of the structures inscribed in glass by ultrafast lasers [2]. Thermal stability of the femto-inscribed grating is comparable to that of type II, UV-inscribed gratings formed by optical damage. No significant difference was established between the observed annealing behavior of the structures directly written by a tightly focused ultrafast laser beam and the reported earlier hightemperature tests of the gratings, produced with a phase mask by a defocused beam of a similar laser.

In conclusion, thermal annealing of fiber Bragg gratings, produced by direct, point-bypoint femtosecond writing has been investigated for the first time. The gratings were formed in commercial fibers without any photosensitization. The gratings were thermally stable up to temperatures of the order of $1000^{\circ} \mathrm{C}$, showing a significant improvement compared to the conventional, UV inscribed FBGs. Dynamics of reflectivity during the annealing-cooling cycles indicates, in particular, that the strain in the material volume adjacent to the modified region is a significant factor affecting the grating performance.

\section{Acknowledgments.}

The authors acknowledge valuable assistance by Mr M. Dubov. and PC Won

\section{References}

1 Y. Kondo, K. Nouchi, T. Mitsuyu, M. Watanabe, P. G. Kazansky, and K. Hirao, "Fabrication of long-period fiber gratings by focused irradiation of infrared femtosecond laser pulses" Opt. Lett. 24, 646-648 (1999) 
2 D.Grobnic, C. W. Smelser,and S.J. Mihailov, "Isothermal behavior of fibre bragg gratings made with ultrafast laser at temperatures above 1000C", ECOC 2004 Proceedings, paper Tu1.3.5.

3 A Martinez, M. Dubov, I.Y. Khrushchev, I. Bennion, "Direct writing of fibre Bragg gratings by femtosecond laser", Electron. Lett. 40, 1170-1172 (2004).

4 A. Othonos and K. Kalli "Fibre Bragg Gratings: Fundamentals and Applications in Telecommunications and Sensors", Artech House Books, (1999)

5 A. M. Strelsov and N. F. Borrelli, "Study of femtosecond laser written waveguides in glasses", J. Opt. Soc. Am. B, 19, (2002), 2496-2504.

6 H.B. Sun, Y. Xu, S. Juodkazis, K. Sun, M. Watanabe, S. Matsuo, H. Misawa and J. Nishii, "Arbitrary-lattice photonic crystals created by multiphoton microfabrication" Opt. Lett. 26, 325-327 (2001))

7 AD Kersey, TA Berkoff and WW Morey, "High-resolution fibre-grating based strain sensor with interferometric wavelength shift detection”, Electron. Lett. 28, (1992), 236-238.

\section{Authors' affiliations:}

Amós Martinez, Igor Y Khrushchev, lan Bennion

Photonics Research Group, Aston University, Birmingham B4 7ET, UK

\section{Figure captions:}

Figure 1. Reflection and transmission spectra of a second order grating inscribed in standard telecommunication fiber.

Figure 2. Isothermal evolution of reflection of femto-inscribed and UV-inscribed gratings during a) 24-hour annealing and b) 10 hours of cooling down.

Figure 3. Spectral evolution of FBG during thecooling down periodafter annealing at $700^{\circ} \mathrm{C}$. Dotted line - Black trace shows the original spectrum before the annealing. 
Figure 4. Dynamics of reflection in a femto-inscribed FBG during annealing at increasing temperatures. 
Figure1.

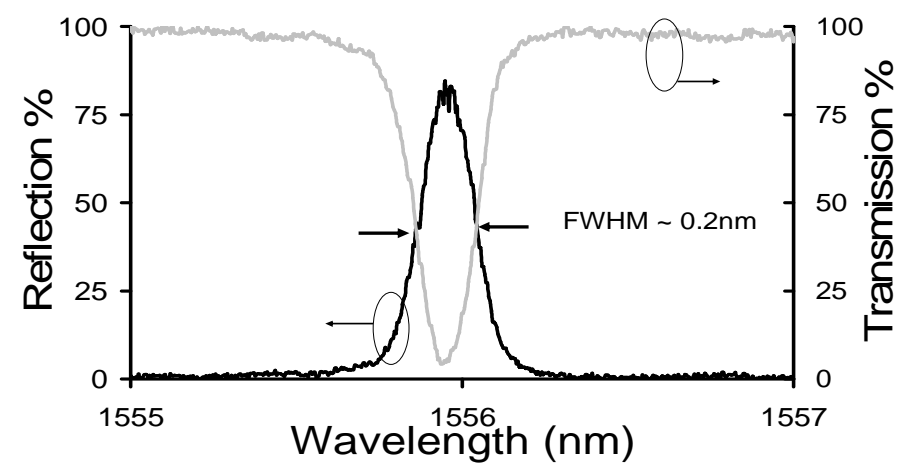


Figure 2.
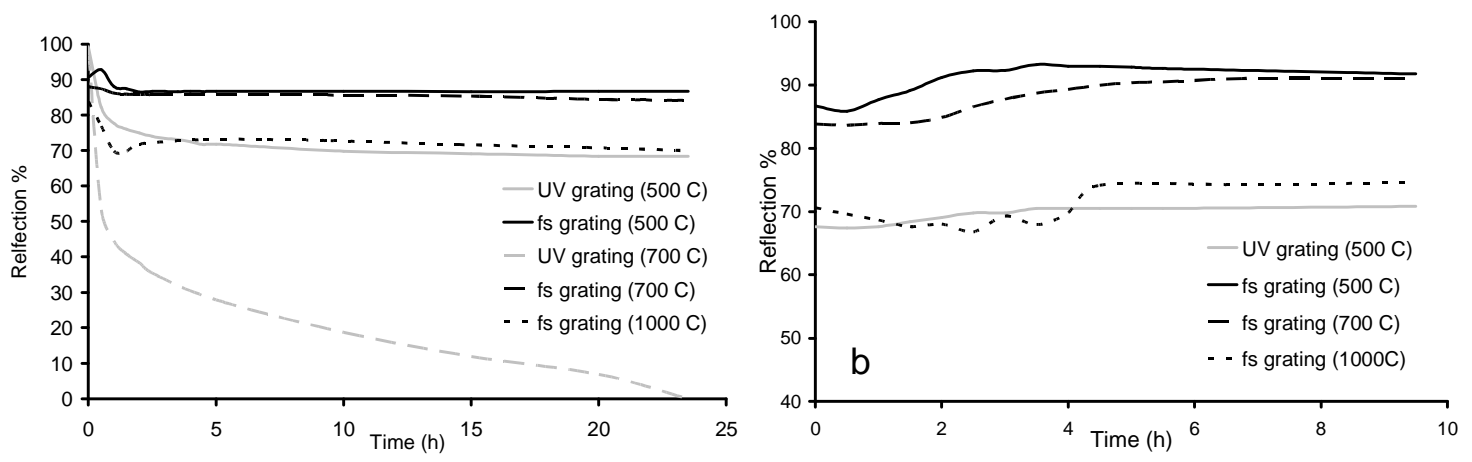
Figure 3.

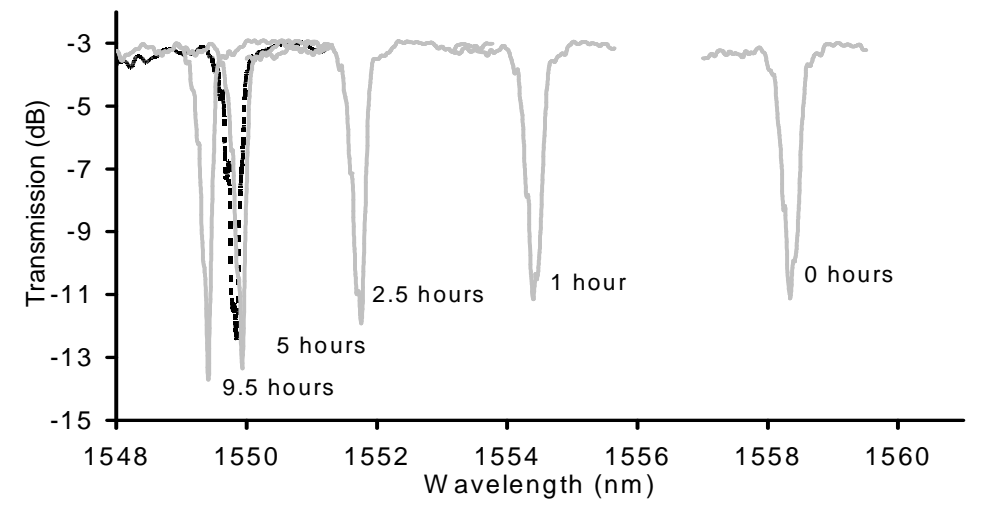


Figure 4.

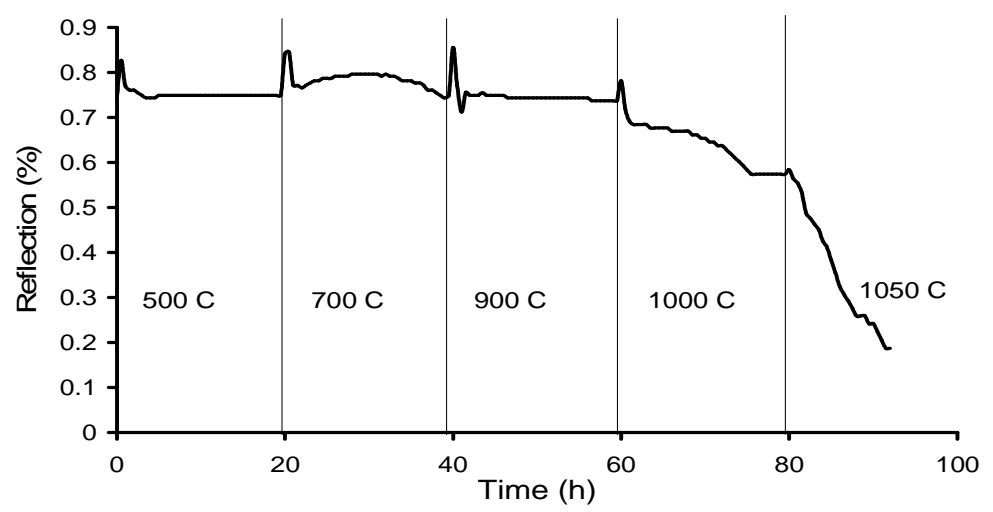

